\title{
Hermite- Padé projection to thermal radiative and variable conductivity MHD flows through channel with a sliding wall
}

\author{
Md. S. Alam ${ }^{1 *}$, M.A.H. Khan ${ }^{2}$ \\ ${ }^{1}$ Department of Mathematics, Jagannath University, Dhaka-1100, BANGLADESH \\ ${ }^{2}$ Department of Mathematics, Bangladesh University of Engineering and Technology, Dhaka-1000, BANGLADESH \\ "Corresponding Author: e-mail: sarwardu75@gmail.com, Tel +8801819298780, Fax+88-02-7113752
}

\begin{abstract}
The combined effect of variable thermal conductivity and radiative heat transfer on steady flow of a conducting optically thin viscous fluid through a channel with sliding wall and non-uniform wall temperatures under the influence of an externally applied homogeneous magnetic field are analyzed in the present study. The similarity transformation reduces the time dependent governing equations for momentum and thermal energy into a set of coupled ordinary differential equations which are solved using perturbation method together with Hermite- Padé approximation. The velocity and temperature profiles are presented graphically to interpret the effect of various physical parameters of the problem. The critical relationships among the parameters are also performed qualitatively.
\end{abstract}

Keywords: Thermal radiation, variable thermal conductivity, sliding wall, non-uniform wall temperature, Hermite- Padé approximation.

DOI: http://dx.doi.org/10.4314/ijest.v6i1.10

\section{Introduction}

The flow and heat transfer between parallel plates channel has been studied by a number of authors (Arpaci et al, 2000), (Makinde, 2008), (Sahin, 1999) and occur in many technological applications, such as in biomedical engineering, material processing, as well as the food and petro-chemical industries. Thermal radiation always exits and can strongly interact with convection in many situations of engineering interest.

Convection in a channel in the presence of thermal radiation draw the attention because of its importance in many practical applications like a furnace, combustion chamber, cooling tower, rocket engine, and solar collector (Chang et al, 1983). However, radiative heat transfer has a key impact in high temperature regime. Many technological processes occur at high temperature and good working knowledge of radiative heat transfer plays an instrumental role in designing the pertinent equipment. (Makinde and Mhone, 2005) considered heat transfer to MHD oscillatory flow in a channel filled with porous medium. In (Cogley et al, 1968), the differential approximation for radiative heat transfer in a nonlinear equation for gray gas near equilibrium was proposed. (Chawla and Chan, 1980) studied the effect of radiation heat transfer on thermally developing Poiseuille flow with scattering. The interaction of thermal radiation with conduction and convection in thermally developing, absorbing-emitting, non-gray gas flow in a circular tube was investigated by (Tabanfar and Modest, 1987). (Choudhury and Das, 2012) have extended the problem (Makinde and Mhone, 2005) to the case of viscoelastic fluid characterized by second-order fluid. (Makinde, 2008) studied the steady state solutions for viscous reactive flows through channels with a sliding wall. The steady-state solutions of a strongly exothermic reaction of a viscous combustible material in a channel filled with a saturated porous medium under Arrhenius kinetics using brinkman model analysed by (Makinde, 2006). The thermal conductivity of the fluid had been assumed to be constant in all the above studies. However, it is known that this physical property may be change significantly with temperature. For a liquid, it has been found that the thermal conductivity $\mathrm{k}$ varies with temperature in an approximately linear manner in the range from 0 to 
$400^{\circ} \mathrm{F}$ (Kay, 1966).

Meanwhile, the thermal boundary layer equation for variable conductivity fluid in the presence of thermal radiation composes a nonlinear problem. The theory of nonlinear differential equations is quite elaborate and their solution remains an extremely important problem of practical relevance in science and engineering. In the last few decades quite a few numerical methods have developed, e. g. finite difference, spectral method, shooting method, etc to tackle this type of problem. Moreover, the models on classical semi-analytical methods have experienced a revival, in connection with the scheme of new hybrid numerical-analytical techniques for nonlinear differential equations, such as Hermite-Padé Approximation Method, which demonstrated itself as a powerful benchmarking tool and a prospective substitute to traditional numerical techniques in various applications in science and engineering. (Makinde, 2009) investigated the variable viscosity and thermal radiation effects on entropy generation rate, the problem of inherent irreversibility in the flow of a temperature dependent variable viscosity optically thin fluid through a channel with isothermal walls studied using Hermite-Padé semi-analytical approach. (Yasir et al, 2011) analyzed the effects of variable viscosity and thermal conductivity on the flow and heat transfer in a laminar liquid film on a horizontal stretching sheet. (Paresh and Archana, 2010) studied that thermal radiation effects on steady boundary-layer flow with variable thermal conductivity over a non-isothermal stretching sheet placed at the bottom of a saturated porous medium. (Pinarbasi et al, 2011) investigates the effect of variable viscosity and thermal conductivity of a nonisothermal, incompressible Newtonian fluid flowing under the effect of a constant pressure gradient at constant temperatures in plane Poiseuille flow using Chebyshev pseudospectral method. (Sadik et al, 2011) studied the effect of variable thermal conductivity and viscosity on single phase convective heat transfer in slip flow.

In view of the above analysis, the aim of the present work is to study the effect of thermal radiation on viscous temperature dependent conductivity flows through a channel with sliding wall under the influence of an externally applied homogeneous magnetic field with the help of perturbation method along with Hermite-Padé approximation. It is assumed that thermal conductivity has a linear variation with temperature. To the best of our knowledge the study undertaken here has not been reported so far by any investigator. It has, therefore, prompted us to investigate heat transfer with variable thermal conductivity and radiation aspects in flow due to sliding wall with non-uniform temperature through a channel.

\section{Mathematical formulation}

Consider a steady two-dimensional laminar incompressible flow of conducting optically thin viscous fluid through a channel with the lower sliding wall and non-uniform wall temperatures under the influence of an externally applied homogeneous magnetic field and radiative heat transfer. Assume that the fluid with negligible absorption has small electrical conductivity and the electromagnetic force produced is very small. A Cartesian coordinate system is used and the flow is chosen along the x-direction under constant pressure-gradient which is driven solely by uniform velocity at the lower wall, i.e. the velocity profile is linear with zero at the upper fixed wall and maximum value at the lower moving wall.

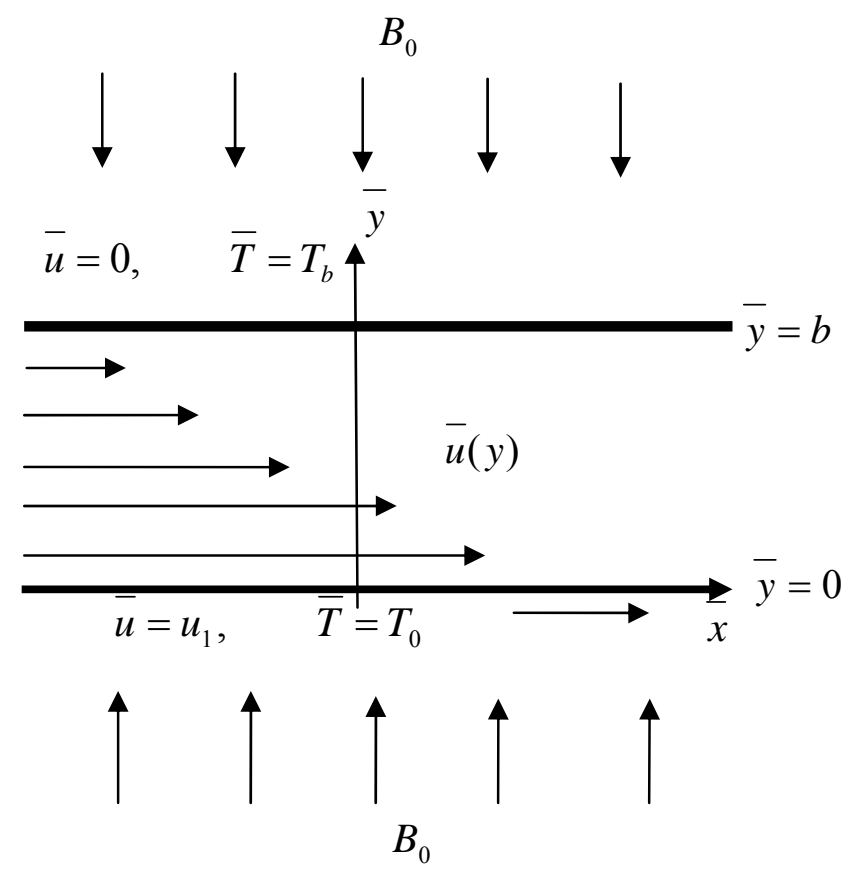

Figure1 Geometry of the problem 
Consider all the physical properties of the fluid constant except the thermal conductivity which varies linearly with temperature, assuming Boussinesq approximation for radiative heat flux and neglecting the viscous dissipation in the energy equation, the boundary-layer equations are

$$
\begin{aligned}
& v \frac{d^{2} \bar{u}}{d \bar{y}^{2}}-\frac{1}{\rho} \frac{d p}{d \bar{x}}-\frac{\sigma_{e} B_{0}^{2}}{\rho} \bar{u}+g \beta\left(\bar{T}-T_{0}\right)=0 \\
& \frac{\bar{k}}{\rho c_{p}} \frac{d^{2} \bar{T}}{d \bar{y}^{2}}-\frac{1}{\rho c_{p}} \frac{d q}{d \bar{y}}=0
\end{aligned}
$$

Where $\bar{u}$ is the flow velocity in the $x$-direction, $v$ is the kinematic viscosity, $\bar{T}$ is the temperature, $\bar{k}$ is the thermal conductivity which is assumed to be variable, $\rho$ is the density of the fluid, $p$ the constant pressure, $c_{p}$ is specific heat at constant pressure, $g$ the gravitational force, $q$ the radiative heat flux, $\beta$ the coefficient of volume expansion due to temperature, $B_{0}=\left(\mu_{e} H_{0}\right)$ the electromagnetic induction, $\mu_{e}$ the magnetic permeability, $H_{0}$ the intensity of magnetic field, $\sigma_{e}$ the conductivity of the fluid The appropriate boundary conditions of the problem are

$$
\begin{array}{ll}
\bar{u}=u_{1}, \quad \bar{T}=T_{0} & \text { at } \bar{y}=0, \\
\bar{u}=0, \quad \bar{T}=T_{b} & \text { at } \bar{y}=b
\end{array}
$$

It is assumed that the medium is optically thin and with relatively low density. Following (Cogley et al, 1968) equilibrium model, the radiative heat flux is given by

$$
\frac{d q}{d \bar{y}}=4 \gamma^{2}\left(T_{0}-T_{b}\right)
$$

Where $\gamma$ is the mean radiation absorption coefficient. The dimensionless temperature $T$ is given by, $T=\frac{\bar{T}-T_{0}}{T_{b}-T_{0}}$

Following (Kay, 1966), the variable thermal conductivity $\bar{k}=k_{\infty}[1+\varepsilon T]$

Where $k_{\infty}$ is the thermal conductivity at the fluid ambient temperature $T_{0}$ and $\varepsilon$ is defined by $\varepsilon=\frac{1}{\bar{k}}\left(\frac{\partial \bar{k}}{\partial T}\right)$.

Let $N=\frac{1}{\mu} \frac{d p}{d \bar{x}}$ be a constant axial pressure gradient with constant viscosity.

Dimensionless quantities and parameters are initiated in the problem as follows

$$
\begin{aligned}
& \eta=\frac{\bar{y}}{b}, \quad \tau=\frac{\bar{x}}{b}, \quad u=\frac{\bar{u}}{u_{1} N}, \quad k=\frac{\bar{k}}{k_{\infty}}, P=\frac{b \bar{P}}{\rho v u_{1}}, \alpha=\varepsilon T \\
& \bar{R}=\frac{4 \gamma^{2} b^{2}}{k_{\infty}}, \bar{H}=\sqrt{\frac{b^{2} \sigma_{e} B_{0}^{2}}{\mu}}, G r=\frac{g \beta\left(T_{b}-T_{0}\right) b^{2}}{v u_{1}},
\end{aligned}
$$

where $\alpha, \bar{R}, \bar{H}$, and $G r$ are thermal conductivity variation parameter, radiation parameter, Hartmann number and Grashof number respectively.

The governing equations (1) and (2) together with boundary conditions (3) and (4) reduced to the following dimensionless form

$$
\begin{aligned}
& \frac{d^{2} u}{d \eta^{2}}-\bar{H}^{2} u+G r T=N, \quad \frac{d^{2} T}{d \eta^{2}}+\alpha T \frac{d^{2} T}{d \eta^{2}}-\bar{R} T=0 \\
& \frac{d^{2} u}{d \eta^{2}}-\alpha H^{2} u+G r T=N \\
& \frac{d^{2} T}{d \eta^{2}}+\alpha\left[T \frac{d^{2} T}{d \eta^{2}}-R T\right]=0
\end{aligned}
$$

Where $k=1+\alpha T, R=\frac{\bar{R}}{\alpha}, H^{2}=\frac{\bar{H}^{2}}{\alpha}$,

With

$u=0, \quad T=1$, at $\quad \eta=1$ 
$u=1, \quad T=0$, at $\quad \eta=0$

\section{Perturbation analysis}

The following power series expansions are considered in terms of the parameter $\alpha$ as equations (9) and (10) are non-linear for velocity field and temperature distribution

$$
u=\sum_{i=0}^{\infty} u_{i} \alpha^{i}, \quad T=\sum_{i=0}^{\infty} T_{i} \alpha^{i}
$$

The non-dimensional governing equations are then solved into series solutions by substituting the Eq.(13) into Eqs. (9) and (10) and equating the coefficients of powers of $\alpha$.

Order zero $\left(\alpha^{0}\right)$

$$
\begin{array}{lr}
\frac{d^{2} u_{0}}{d \eta^{2}}+G r T_{0}=N, & \frac{d^{2} T_{0}}{d \eta^{2}}=0 \\
u_{0}=0, T_{0}=1 & \text { at } \eta=1 \\
u_{0}=1, \quad T_{0}=0 & \text { at } \eta=0
\end{array}
$$

Order one $\left(\alpha^{1}\right)$

$$
\begin{array}{ll}
\frac{d^{2} u_{1}}{d \eta^{2}}-H^{2} u_{0}+G r T_{1}=N, & \frac{d^{2} T_{1}}{d \eta^{2}}+T_{0} \frac{d^{2} T_{0}}{d \eta^{2}}-R T_{0}=0 \\
u_{1}=0, T_{1}=0 & \text { at } \eta=1 \\
u_{1}=0, \quad T_{1}=0 & \text { at } \eta=0
\end{array}
$$

With the help of MAPLE, we have computed the first 18 coefficients for the series of the velocity $u$ and temperature field $T$ as well as the series for the wall heat transfer rate, $N u=-\frac{d T}{d \eta}$ at $\eta=1$ in terms of $\alpha, H, R, G r, N$. The first few coefficients of the series for $u$ and $T$ are as follows:

$$
\begin{aligned}
& u(\eta, \alpha, H, R, G r, N)=-\frac{1}{6}(\eta-1)\left(G r \eta^{2}-3 \eta N+G r \eta+6\right)-\frac{1}{360} \eta(\eta-1)\left(3 H^{2} G r \eta^{3}+3 G r R \eta^{3}-15 H^{2} \eta^{2} N+3 \eta^{2} H^{2} G r+3 \eta^{2} G r R\right. \\
& \left.-7 G r R \eta+15 H^{2} \eta N-7 H^{2} G r \eta+60 H^{2} \eta-7 H^{2} G r-120 H^{2}-180 N+15 H^{2} N-7 G r R\right) \alpha+O\left(\alpha^{2}\right) \\
& T(\eta, \alpha, R, H, G r, N)=\eta+\frac{1}{6} R \eta(\eta-1)(\eta+1) \alpha+\frac{1}{360} R \eta(\eta-1)\left(3 \eta^{3} R-30 \eta^{2}+3 \eta^{2} R-7 \eta R-30 \eta-7 R-30\right) \alpha^{2}+O\left(\alpha^{3}\right)
\end{aligned}
$$

\section{Computational procedure}

In the present analysis, we shall employ a very efficient solution method, known as Hermite-Padé approximants, which was first introduced by (Padé, 1892) and (Hermite, 1893). All the one variable approximants that are used or discussed throughout this article belong to the Hermite-Padé class. In its most general form, this class is concerned with the simultaneous approximation of several independent series.

For any non-negative integer $d$, let the $d+1$ power series $u_{0}(\eta), u_{1}(\eta), \ldots \ldots \ldots . . ., u_{d}(\eta)$ are given. Assume that the $(d+1)$ tuple of polynomials: $P_{N}^{[0]}, P_{N}^{[1]}$, ,$P_{N}^{[d]}$

where $\operatorname{deg} P_{N}^{[0]}+\operatorname{deg} P_{N}^{[1]}+\ldots+\operatorname{deg} P_{N}^{[d]}+d=N$,

is a Hermite-Padé form of these series if $\sum_{i=0}^{d} P_{N}^{[i]}(\eta) u_{i}(\eta)=O\left(\eta^{N}\right)$ as $\eta \rightarrow 0$. 
Here $u_{0}(\eta), u_{1}(\eta), \ldots \ldots \ldots \ldots, u_{d}(\eta)$ may be independent series or different form of a unique series. We need to find the polynomials $P_{N}^{[i]}$ that satisfy the equations (22) and (23). These polynomials are completely determined by their coefficients. So, the total number of unknowns in equation (23) is $\sum_{i=0}^{d} \operatorname{deg} P_{N}^{[i]}+d+1=N+1$

Expanding the left hand side of equation (23) in powers of $\eta$ and equating the first $N$ equations of the system equal to zero, we get a system of linear homogeneous equations. To calculate the coefficients of the Hermite-Padé polynomials it requires some sort of normalization, such as $P_{N}^{[i]}(0)=1$ for some integer $0 \leq i \leq d$

It is important to emphasize that the only input required for the calculation of the Hermite-Padé polynomials are the first $N$ coefficients of the series $u_{0}(\eta), u_{1}(\eta), \ldots \ldots \ldots \ldots, u_{d}(\eta)$. The equation (24) simply ensures that the coefficient matrix associated with the system is square. One way to construct the Hermite-Padé polynomials is to solve the system of linear equations by any standard method such as Gaussian elimination or Gauss-Jordan elimination. (Drazin -Tourigney, 2000) Approximants is a particular kind of algebraic approximants and (Khan, 2002) introduced High-order differential approximant as a special type of differential approximants. High-order partial differential approximants discussed in (Rahman, 2004) is a multivariable differential approximants. More information about the above mentioned approximants can be found in the respective references. An algebraic programming language MAPLE is used to compute the series coefficients of non-dimensional governing equation of the problem.

\section{Results and Discussions}

The main objective of the current work is to analyze the effect of thermal radiation and thermal conductivity variation due to temperature on MHD flow of viscous incompressible optically thin fluid through a channel with the lower sliding wall. Although there are four parameters of interest in the present problem the effects of thermal conductivity variation parameter $\alpha$, radiation parameter $R$, Hartman number $\mathrm{Ha}$ and Grashof number $\mathrm{Gr}$.

The results of the numerical computations of velocity profiles and temperature distributions for different values of the aforementioned parameters are displayed graphically in Figures. (2)-(7) by analyzing the series in (20) and (21) using HermitePadé approximation method.

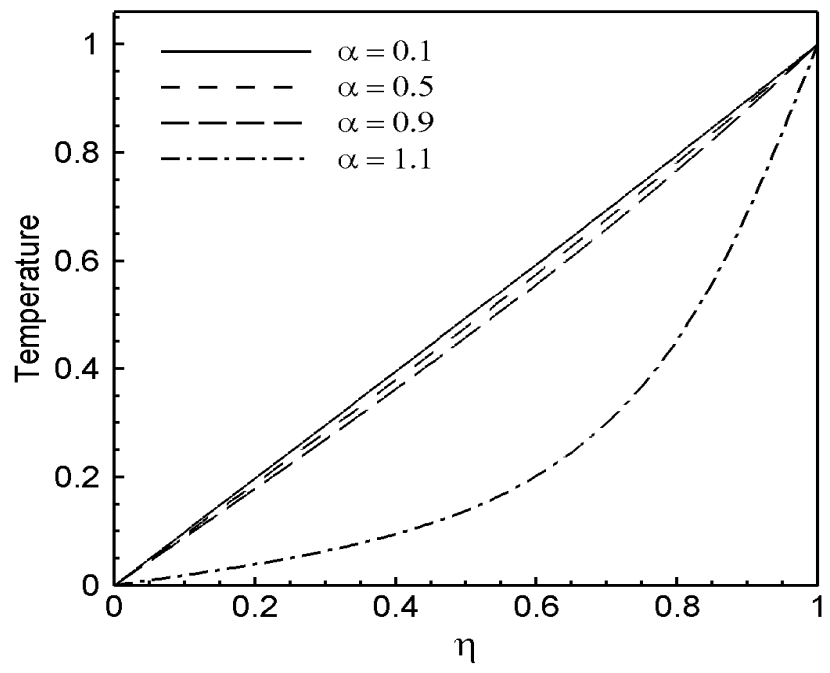

(a)

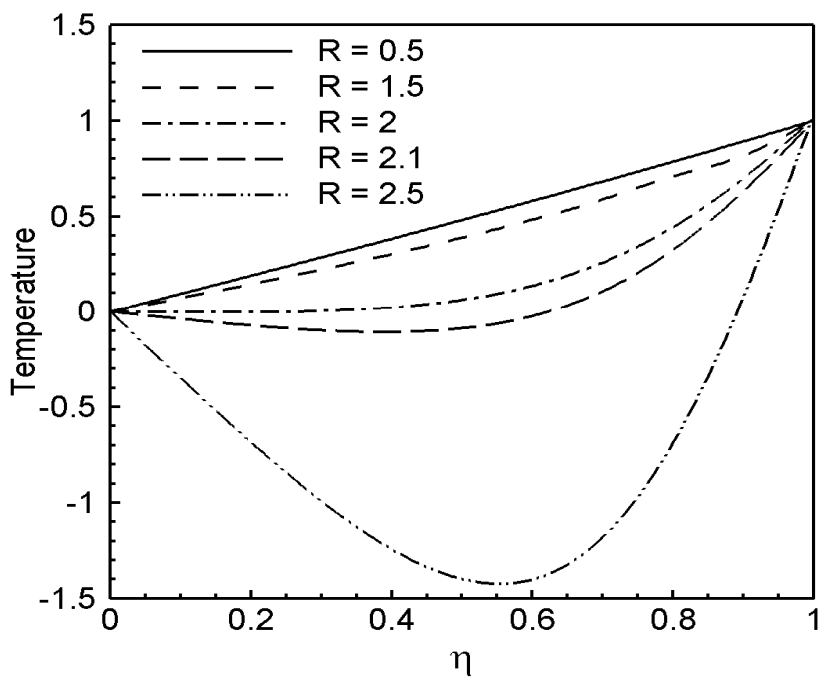

(b)

Figure 2 Temperature distribution (a) for different values of $\alpha$ at $\mathrm{R}=1, \mathrm{~N}=1, \mathrm{H}=1, \mathrm{Gr}=1$ and (b) for different values of $R$ at $\alpha=0.9, \mathrm{~N}=1, \mathrm{H}=1, \mathrm{Gr}=1$ using High-order differential approximants (2002).

Figures 2 (a)-(b) illustrate the effects of thermal conductivity variation parameter $\alpha$ and thermal radiation parameter $R$ on the temperature profiles respectively. It is observed from the Figure 2(a) that an increase in the thermal conductivity variation parameter $\alpha$ leads to decrease in the temperature across the center line region particularly for $\alpha>1$. We also observe from Figure 2(b) that the fluid temperature decreases with the increasing values of $R$. Because the increasing values of $\alpha$ and $R$ decrease the temperature differences between the surface and outside the boundary layer. Then heat is transferred slowly from surface to fluid within the boundary layer. That's why temperature distributions decrease with the increasing values of $\alpha$ and $R$. Meanwhile, the 
minimum temperature is generally seen along the channel centerline and then increases gradually to the prescribed value at the upper wall. Figures (3)-(7) reveal the velocity profiles for increasing values of thermal conductivity variation parameter $\alpha$, thermal radiation parameter $R$, axial pressure gradient parameter $N$, magnetic parameter $H$ and Grashof number $G r$. A parabolic velocity profile is observed generally in Figures (3) and (4) with maximum value at the lower wall and minimum value along the channel centerline region. It is clear that an increasing presence of $\alpha$ and $R$ gives a reduction in the fluid velocity as demonstrated particularly along the centerline, i.e. the reverse flow takes place near the centerline because the influence of $\alpha$ and $R$ surpasses the action of the viscous force in that region which specially coincides with generalized Plane Couette flow. From Figure (5), it is noticed that the velocity profile exhibits major changes along the centerline region at every increasing values of $\mathrm{N}$.

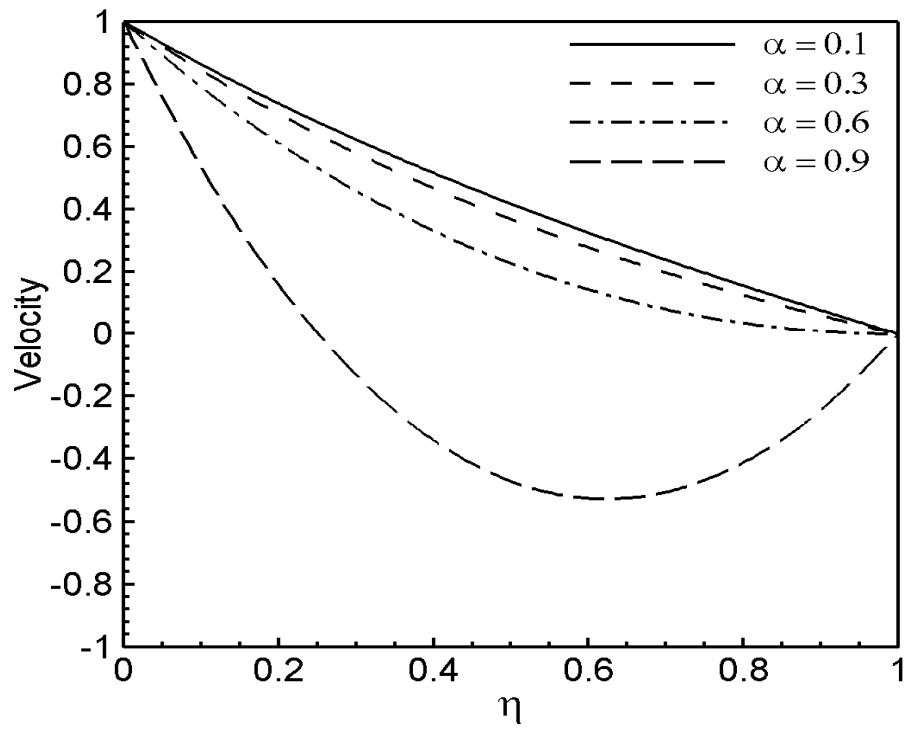

Figure 3 Velocity profile for different values of $\alpha$ at $\mathrm{R}=1, \mathrm{~N}=1, \mathrm{H}=1, \mathrm{Gr}=1$ using High-order differential approximants (2002).

On the other hand, it is seen from Figure (6) an uniform decreases in the centerline velocity at the positive variation of Hartman number $H$. The magnetic field acting along the horizontal direction retards the fluid velocity. Applied magnetic field creates a Lorentz force by the interaction between magnetic field and flow field, this force acts against the fluid flow and reduce the velocity distribution. Moreover, there is a minor variation is seen to the fluid velocity in Figure (7) due to the effect of Grashof number Gr.

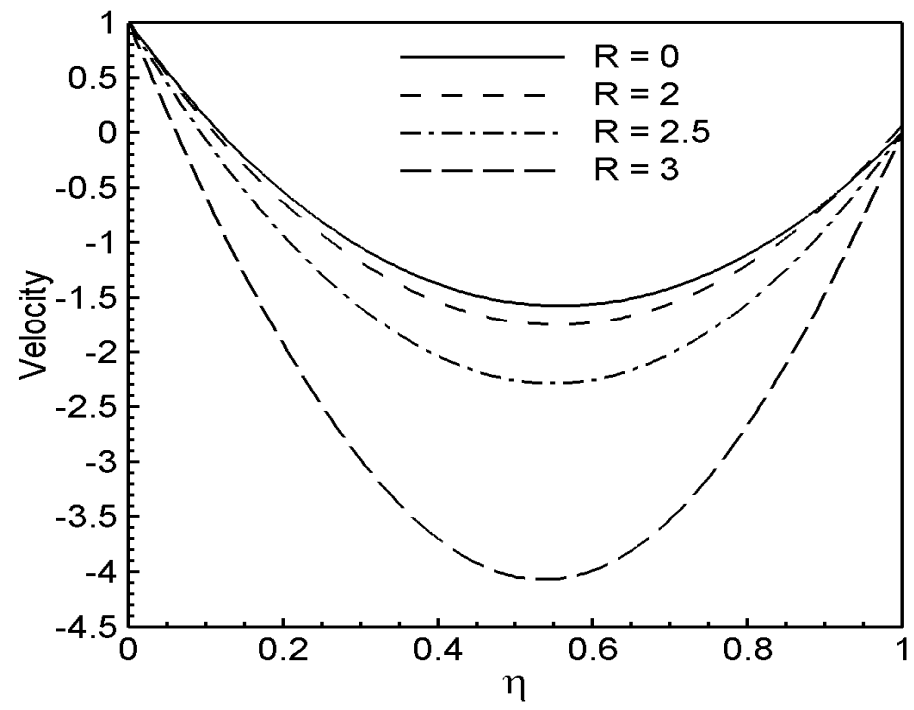

Figure 4 Velocity profile for different values of $R$ at $\mathrm{H}=1, \mathrm{~N}=1, \alpha=1, \mathrm{Gr}=1$ using High-order differential approximants (2002). 


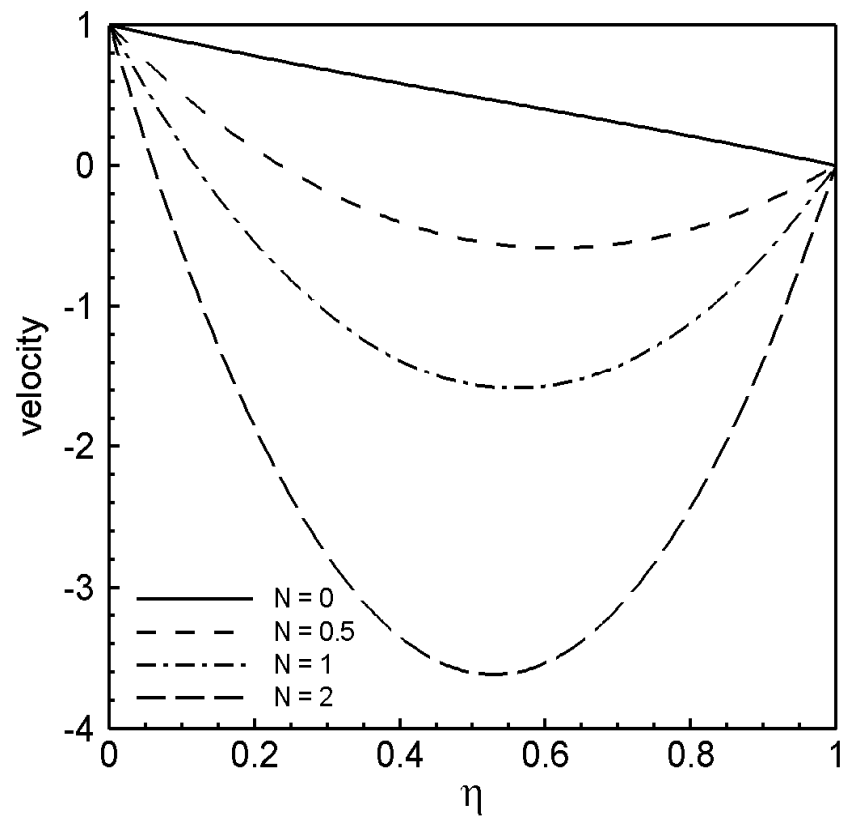

Figure 5 Velocity profile for different values of $N$ at $\mathrm{H}=1, \mathrm{R}=1, \alpha=1, \mathrm{Gr}=1 \mathrm{using}$ High-order differential approximants (2002).

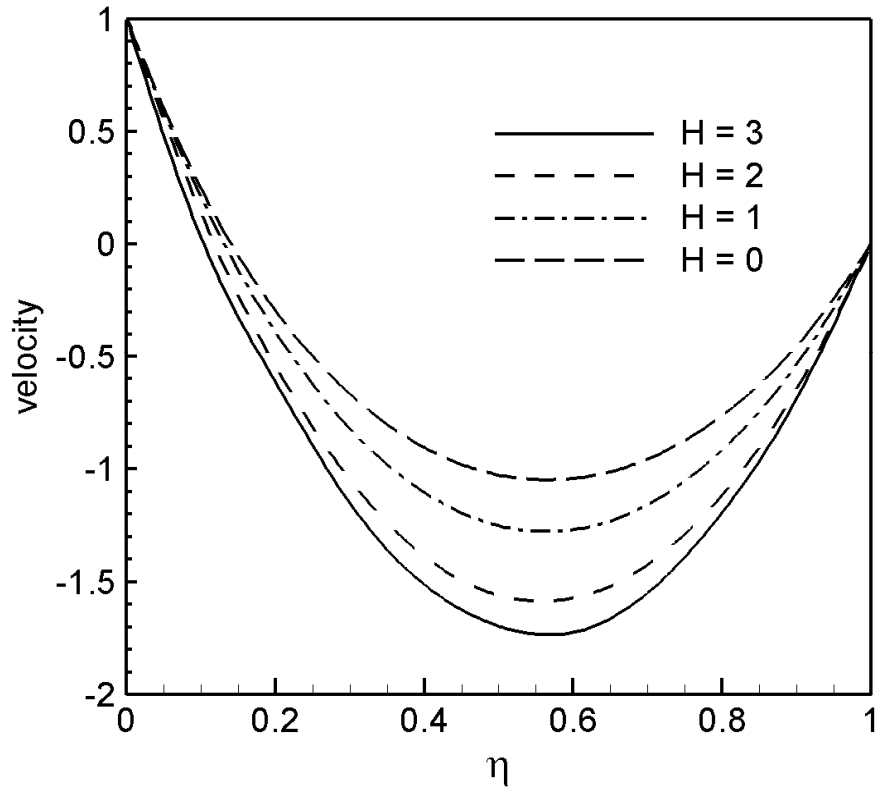

Figure 6 Velocity profile for different values of $H$ at $\mathrm{N}=1, \mathrm{R}=1, \alpha=1, \mathrm{Gr}=1$ using High-order differential approximants (2002). 


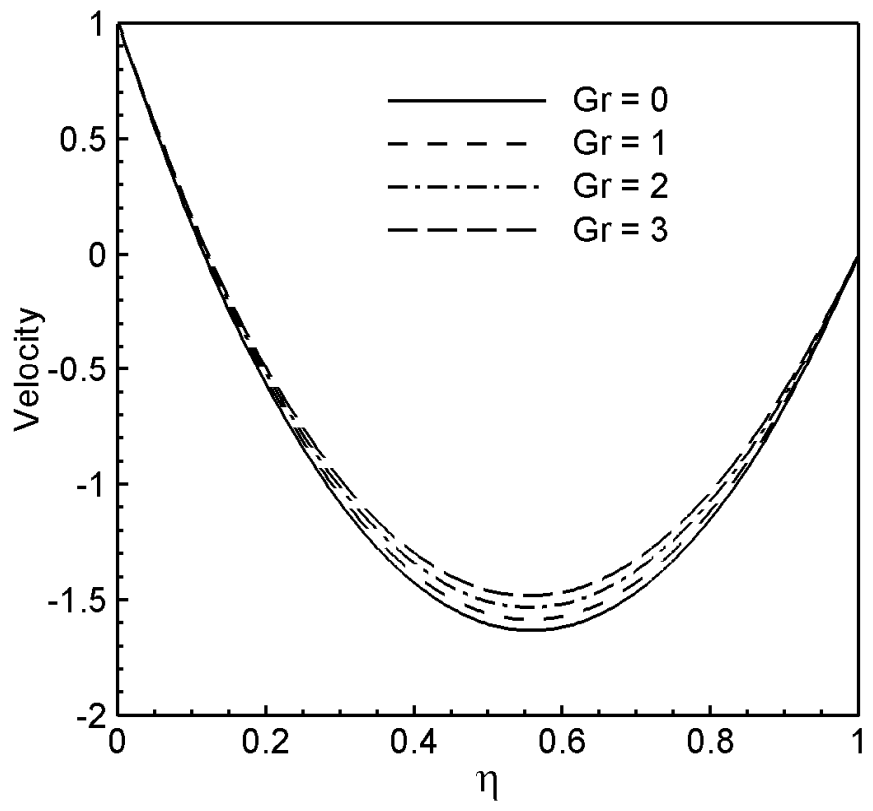

Figure 7 Velocity profile for different values of $G r$ at $\mathrm{N}=1, \mathrm{R}=1, \alpha=1, \mathrm{H}=1$ using High-order differential approximants (2002).

Applying high-order partial differential approximate method to the series (21) the critical relationship among the parameters of the solution is analyzed with the influence of $R$ and $\alpha$. Figure 8 shows the critical relationship between $\alpha$ and $N u$ for $d=4$ and the curves in this figure really increase due to the effect of increasing values of $R$. It is also noticed that for $R \geq 1$, there occurs an abrupt change in the curve. Figure 9 displays the effect of thermal conductivity variation parameter $\alpha$ on the critical relation between $R$ and $N u$ with the same order of approximation. It is interesting to see that as $\alpha$ increases $N u$ increases, but the value of $N u$ decreases rapidly at $0 \leq R \leq 1$ and for $R>1, N u$ increases slowly due to the raising values of $R$. This means that the thermal conductivity variation parameter $\alpha$ acts like a control parameter to the relationship between $R$ and $N u$.

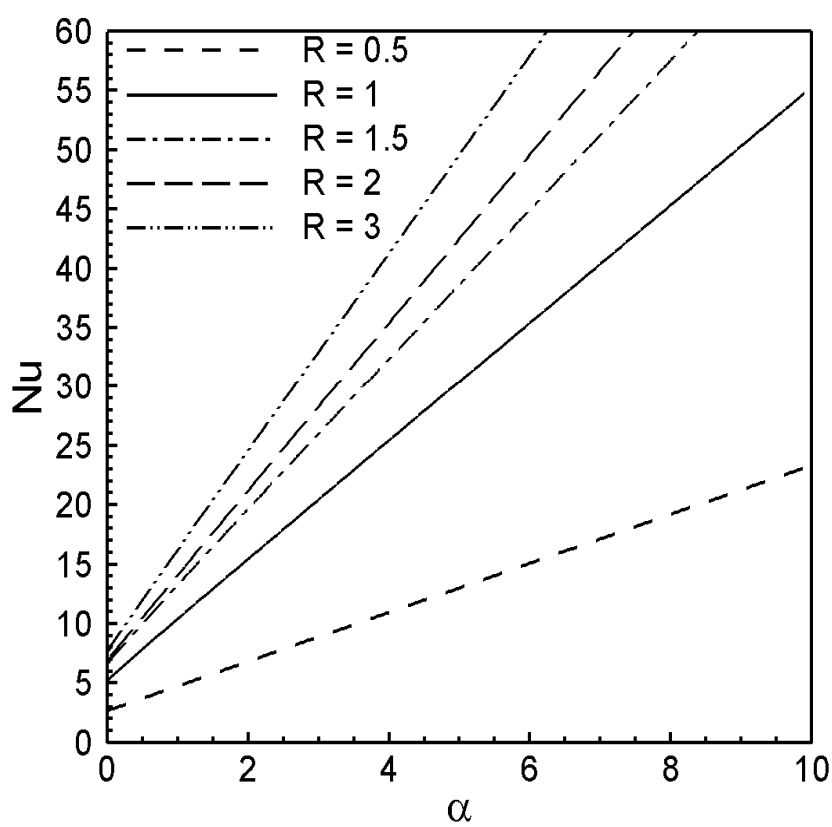

Figure 8 Relation between $N u$ and $\alpha$ for different values of $R$ using High-order partial differential approximants (2004). 


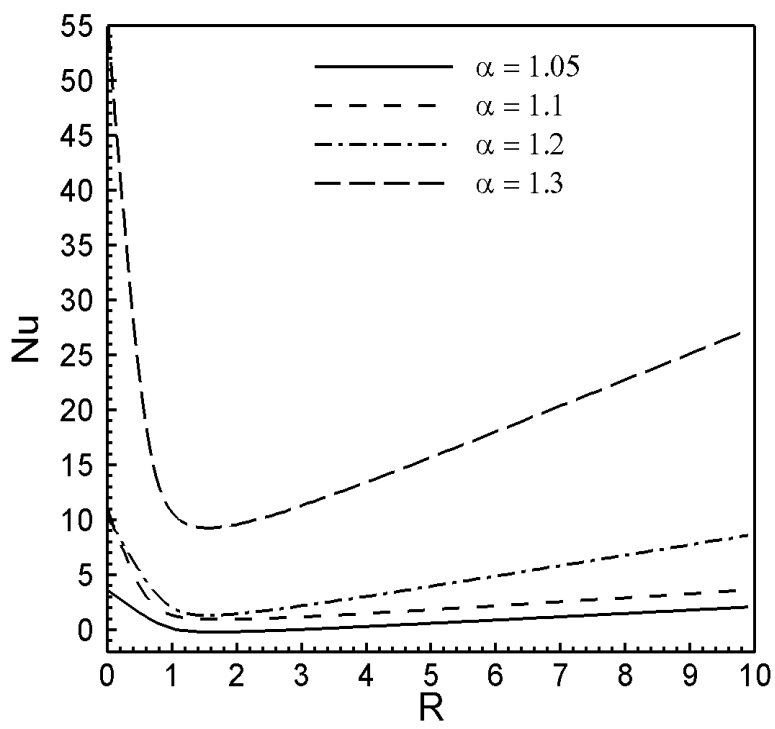

Figure 9 Relation between $N u$ and $R$ for different values of $\alpha$ using High-order partial differential approximants (2004).

\section{Conclusion}

The influences of thermal radiation on MHD flow of an optically thin variable thermal conductivity viscous incompressible fluid through a channel with the lower sliding wall of non-uniform temperatures are investigated using a special type of Hermite-Padé approximation technique. The velocity and temperature profiles are obtained analytically to observe the effect of the parameters of the solution. The escalating values of thermal radiation and thermal conductivity reduce centerline velocity while the increasing magnetic parameter causes a reduction in the magnitude of centerline velocity field. The relationship between Nusselt number and thermal conductivity variation parameter varies due to the effect of radiation parameter whereas the relationship between Nusselt number and radiation parameter varies due to the effect of thermal conductivity variation parameter. Moreover, we provide a basis for guidance about new approximants idea for summing power series that should be chosen for many problems in fluid mechanics and similar subjects.

\section{Acknowledgements}

This work was done within the framework the PhD program of the corresponding author under Department of Mathematics, Bangladesh University of Engineering and Technology, Dhaka. Financial support from the Bangabandhu Fellowship on Science and ICT project is acknowledged.

\section{References}

Arpaci V.S., Selamet A. and Kao S.H., 2000. Introduction to Heat Transfer, Prentice-Hall, New York.

Ahmet Pinarbasi, Coskun Ozalp and Selim Duman, 2011. Influence of variable thermal conductivity and viscosity for nonisothermal fluid flow, Physics of Fluids, Vol. 17, No. 3.

Chang L.C., Yang K.T. and Lloyd J.R., 1983. Radiation—natural convection interaction in two-dimensional complex enclosure, J. Heat Transfer, Vol. 105, pp. 89-95.

Cogley A.C.L., Vincenti W.G. and Gilles E.S., 1968. Differential approximation for radiative heat transfer in a nonlinear equations-grey gas near equilibrium, Am. Inst. Aeronaut. Astronaut. J., Vol. 6, pp. 551-553.

Chawla T.C. and Chan S.H., 1980. Combined radiation and convection in thermally developing Poiseuille flow with scattering, $J$.

Heat Transfer, Vol. 102, pp. 297-302.

Choudhury R. and Das U. J., 2012. Heat transfer to MHD oscillatory viscoelastic flow in a channel filled with porous medium, Phys. Res. Int., Vol. 2012, pp. 1-5.

Drazin P.G. and Tourigny Y., 1996. Numerically study of bifurcation by analytic continuation of a function defined by a power series, SIAM Journal of Applied Mathematics, Vol. 56, pp. 1-18.

Hermite C., 1893. Sur la généralisation des fractions continues algébriques., Annali di Mathematica Pura e Applicata, Vol. 21, Sér. 2, pp 289-308. 
Kay W.M., 1966. Convective heat and mass transfer. Mc-Graw Hill, New York.

Khan Y., Wu Q. and Faraz N., 2011. The effects of variable viscosity and thermal conductivity on a thin film flow over a shrinking/stretching sheet, Computers and Mathematics with Applications, Vol. 61, pp.3391-3399.

Khan M.A.H., 2002. High-Order Differential Approximants, Journal of Computational and Applied Mathematics, Vol. 149, pp. 457-468.

Makinde O.D., 2008. Entropy-generation analysis for variable-viscosity channel flow with non-uniform wall temperature, Appl. Energy, Vol. 85, pp. 384-393.

Makinde O.D. and Mhone P.Y., 2005. Heat transfer to MHD oscillatory flow in a channel filled with porous medium, Rom. Journ. Phys., Vol. 50, pp. 931-938.

Makinde O.D., 2008. Thermal criticality in viscous reactive flows through channels with a sliding wall: An exploitation of the Hermite-Padé approximation method, Math. And Comp. Modelling, Vol. 47, pp. 312-317.

Makinde O.D., 2006. Thermal ignition in a reactive viscous flow through a channel filled with a porous medium, ASME, J. Heat Transfer, Vol. 128, pp. 601-604.

Makinde O.D., 2009. Hermite-Padé approach to thermal radiation effect on inherent irreversibility in a variable viscosity channel flow, Comp. and Math. with Applications, Vol. 58, pp. 2330-2338.

Paresh Vyas and Archana Rai, 2010. Radiative flow with variable thermal conductivity over a non-isothermal stretching sheet in a porous medium, Int. J. Contemp. Math. Sciences, Vol. 5, No. 54, pp.2685 - 2698.

Padé H., 1892. (Thesis) Sur la représentation approchée d'une fonction pour des fractions rationnelles., Ann. Sci. École Norm. Sup. Suppl.[3], Vol. 9, pp.1-93.

Rahman M.M., 2004. A New Approach to Partial Differential Approximants, M. Phil. thesis, Bangladesh University of Engineering \& Technology, Dhaka.

Sadık Kakaç, Almıla G. Yazıcıoğlu and Arif Cem Gözükara, 2011. Effect of variable thermal conductivity and viscosity on single phase convective heat transfer in slip flow, Heat and Mass Transfer, Vol. 47, No. 8, pp 879-891.

Sahin A.Z., 1999. Effect of variable viscosity on the entropy generation and pumping power in a laminar fluid flow through a duct subjected to constant heat flux, Heat Mass Transfer, Vol. 35, pp. 499-506.

Tabanfar S. and Modest M.F., 1987. Combined radiation and convection in absorbing, emitting, non-gray gas-particulate tube flow, J. Heat Transfer, Vol. 109, pp. 478-484.

Biographical notes

Md. S. Alam is of the Department of Mathematics, Jagannath University, Dhaka-1100, Bangladesh

M.A.H. Khan is of the Department of Mathematics, Bangladesh University of Engineering and Technology, Dhaka-1000, Bangladesh

Received November 2013

Accepted November 2013

Final acceptance in revised form November 2013 